\title{
Discovering Mathematical Concepts with Dynamic Software: A Demonstration
}

\author{
Sharon Whitton \\ Graduate Director and Professor : Mathematics Education, School of \\ Education and Allied Human Services, 110 Hagedorn Hall, Hofstra \\ University, Hempstead, NY 11549-1190, USA \\ sharon.whitton@hofstra.edu
}

\begin{abstract}
This presentation demonstrates the implementation of dynamic software in secondary mathematics classrooms (grades 7-12). It illustrates successful activities for infusing The Geometer's Sketchpad, version 4.06 (Key Curriculum Press) and Winplot (available free over the internet) into the teaching and learning of geometry, algebra, trigonometry and introductory calculus. Unlike traditional instruction, which follows the lecture $\rightarrow$ example $\rightarrow$ assignment sequence, this software offers modalities for learning that appeal to students with a variety of learning styles. Moreover, it mirrors the recommendations of NCTM and prominent learning theorists. Specifically, NCTM (2000) advocates mathematics instruction that is inquiry-based, collaborative, and involves open-ended investigations. The Geometer's Sketchpad and Winplot provide learning environments that accommodate these activities. Specific applications of this software within an NSFfunded ( $\$ 11.6$ million) project are included. The project, titled "Mathematics, Science and Technology Partnership" (2003-2008), is conducted at Hofstra University on Long Island, New York.
\end{abstract}

